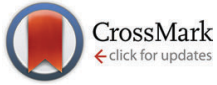

Cite this: Phys. Chem. Chem. Phys., 2015, 17, 18273

Received 12th March 2015,

Accepted 22nd June 2015

DOI: $10.1039 / c 5 c p 01470 k$

www.rsc.org/pccp

\title{
A computational study of the quantum transport properties of a $\mathrm{Cu}-\mathrm{CNT}$ composite $\dagger$
}

\author{
Mahdi Ghorbani-Asl,* Paul D. Bristowe* and Krzysztof Koziol
}

\begin{abstract}
The quantum transport properties of a $\mathrm{Cu}$-CNT composite are studied using a non-equilibrium Green's function approach combined with the self-consistent-charge density-functional tight-binding method. The results show that the electrical conductance of the composite depends strongly on CNT density and alignment but more weakly on chirality. Alignment with the applied bias is preferred and the conductance of the composite increases as its mass density increases.
\end{abstract}

The growing demand for electrical energy, changing weather conditions, rising prices and the need for power in extreme environments all limit the use of conventional conductors in electrical wires and interconnects. Furthermore, power losses cause serious performance issues in these conductors. Novel composite materials containing carbon nanostructures can potentially improve a number of properties of conventional conductors but one important area would be to allow them to transmit current more efficiently and at lower cost. They would also reduce the weight required for a designated transmission line. Carbon nanotubes (CNTs) are obvious candidates for this purpose because of their remarkable physical properties, e.g. high current carrying capacity, ${ }^{1}$ ballistic transport, ${ }^{2}$ low density, and high strength and stiffness. ${ }^{3-5}$ There have already been reports of the promising electrical properties of high quality CNT-based fibres. ${ }^{6,7}$ The fibres have also been used in various electrical applications, e.g. high frequency transformers. ${ }^{8}$ Although CNTs on their own exhibit low free electron density, ${ }^{9}$ this can be addressed by embedding them in transition or simple metals like $\mathrm{Cu}, \mathrm{Cr}, \mathrm{Au}, \mathrm{Ag}$ or $\mathrm{Al}$ to form novel composite materials with high conductivity. ${ }^{10}$ Among these possibilities, $\mathrm{Cu}-\mathrm{CNT}$ composites have attracted particular attention because of their excellent electrical conductivity ${ }^{11}\left(2.3-4.7 \times 10^{5} \mathrm{~S} \mathrm{~cm}^{-1}\right.$ compared to $5.8 \times 10^{5} \mathrm{~S} \mathrm{~cm}^{-1}$ for bulk copper), high thermal conductivity ${ }^{12}\left(395 \mathrm{Wm}^{-1} \mathrm{~K}^{-1}\right.$ compared to $400 \mathrm{Wm}^{-1} \mathrm{~K}^{-1}$ for

Department of Materials Science and Metallurgy, University of Cambridge, Cambridge, CB3 OFS, UK. E-mail: mg741@cam.ac.uk, pdb1000@cam.ac.uk $\dagger$ Electronic supplementary information (ESI) available. See DOI: 10.1039/ c5 cp01470k bulk copper) and low coefficient of thermal expansion (similar to silicon). ${ }^{12}$ In addition, it is found that $\mathrm{Cu}-\mathrm{CNT}$ composites are about three times stronger than pure $\mathrm{Cu}^{13}$ Such composites can be fabricated using various techniques involving powder metallurgy, thermal spraying and electrochemical deposition ${ }^{10}$ although the results show that the electrical conductivity depends strongly on the fabrication process. It is expected that better control over the structural morphology of the CNTs will result in improvements to the conductivity. Computational analysis of the underlying transport properties and microstructure of the composites will guide this process.

First principles calculations on the interaction between copper and CNTs have so far focused on the end or side contact between a nanotube and two copper electrodes. ${ }^{14-17}$ Electron transport across the interface between the CNT and the metal surface is clearly important but the CNT is not embedded within the metal and therefore other transmission pathways cannot occur. More recently, Feng et al. ${ }^{18}$ studied the electrical performance of a $\mathrm{Cu}-\mathrm{CNT}$ matrix using a simple electrical model and concluded that the ratio of aligned CNTs and their dimensions affect the current density distribution. To gain further insight at the microscopic level large-scale quantum transport calculations are necessary. In this work, the electronic structure and transport properties of $\mathrm{Cu}-\mathrm{CNT}$ composites are investigated using a density-functional based approximation method combined with the non-equilibrium Green's function (NEGF) scheme. The effect of CNT density, chirality and alignment to the bias potential is studied. Due to the presence of quantum confinement, each parameter is likely to influence the electrical properties. For the first time a model is constructed in which the CNT is fully embedded within a 3D copper matrix. The results show that the conductance is highest when the long axis of the CNT is parallel to the applied bias. Furthermore the conductance decreases with CNT number density and increases with overall mass density of the composite as expected.

The atomistic model used in the calculations is shown in Fig. 1 and includes a finite central or scattering region, which is connected to two semi-infinite ideal copper electrodes across 


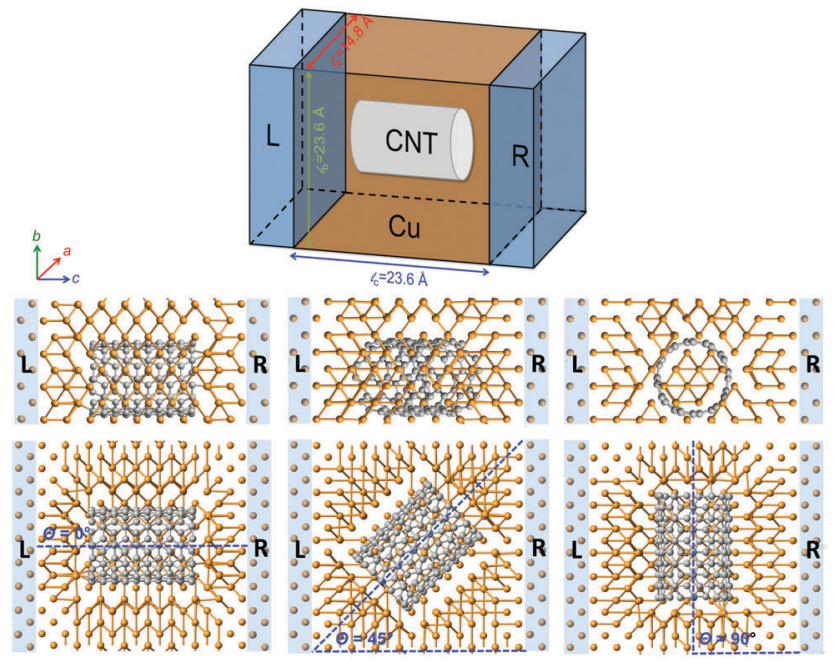

Fig. 1 Upper: Schematic representation of the model used for the Cu-CNT transport calculations. Lower: Optimized structure of a $\mathrm{Cu}-\mathrm{CNT}$ composite containing a $(6,6)$ CNT in three different orientations $(\theta)$ with respect to the transport direction (horizontal $c$-axis). In each case a top and side view is shown. The left and right electrodes $(L$ and $R$ ) consist of semi-infinite perfect copper. Carbon and copper atoms are shown in grey and orange, respectively.

which a bias is applied. In the scattering region the CNT, which is empty, is contained within the $\mathrm{Cu}$ matrix and therefore both the curved surface and ends of the CNT participate in the electron transport. Periodic boundary conditions are applied in all directions perpendicular to the applied bias. Structurally there are many ways to orient the CNT within the metal matrix. In order to maintain symmetry and reduce complexity, three principal configurations are studied with $\theta=0^{\circ}, 45^{\circ}, 90^{\circ}$ where $\theta$ is the angle of inclination of the nanotube axis with respect to the transport direction (the horizontal $c$-axis in Fig. 1). Most of the calculations are performed using a rectangular supercell with dimensions $14.8 \AA$, $23.6 \AA$ and $23.6 \AA$ along $a, b$ and $c$ directions. This corresponds to a CNT number density of $2.87 \times$ $10^{13} \mathrm{~cm}^{-2}$. To investigate composites with different CNT densities the dimensions along $a$ and $b$ are changed. The largest supercell has dimensions $14.8 \times 39.0 \times 23.6 \AA$ corresponding to 1080 atoms and a CNT number density of $1.73 \times 10^{13} \mathrm{~cm}^{-2}$. Both a metallic CNT $(6,6)$ and a semiconducting CNT $(10,0)$ with almost same length $(\sim 11 \AA)$ and very similar diameter $(\sim 8 \AA)$ are considered. This enables a direct comparison of the results for these two cases without the complication of the size dependence. The volume of the $\mathrm{Cu}$ cavity containing the CNT is chosen such that the distance between the $\mathrm{Cu}$ and CNT surfaces agrees with recent density functional theory (DFT) results ${ }^{15,16}$ for optimal separation between the materials, i.e. about 1.9-2.4 Å. For the $\theta=0^{\circ}$ and $90^{\circ}$ orientations, the CNT connects with $\mathrm{Cu}\{100\}$ surfaces at the end contacts whereas for the $\theta=45^{\circ}$ orientation the surfaces are $\{110\}$. To further support this choice of orientations and surface contacts, separate DFT calculations were performed on a $(5,5)$ CNT in contact with three low-index Cu surfaces (see Table S1, ESI $\dagger$ ). A $(5,5)$ CNT is chosen for computational convenience and no significant difference in behaviour is expected if a $(6,6) \mathrm{CNT}$ is used. It is found that the $\{100\}$ orientation is the most stable for both the side and end contacts suggesting they have the lowest energy. The $\{110\}$ end and side contacts are found to be less stable but by no more than $6 \%$ of the ideal work of separation.

All the composite calculations are performed using the density-functional-based tight-binding (DFTB) method $^{19}$ as implemented in the Atomistix ToolKit (ATK). ${ }^{20}$ The structures are optimized applying 3D periodic boundary conditions and total energies are converged to within $10^{-5} \mathrm{eV}$. The Brillouin zone of the whole structure is sampled using $(1 \times 1 \times 100) k$ points. The Poisson equation is solved using a Fast Fourier Transform (FFT) solver. The coherent transport calculations are performed using the DFTB method in conjunction with the NEGF technique. ${ }^{21}$ The calculations involve the self-consistent charge correction (SCC), ${ }^{22}$ which takes into account the electron density redistribution due to interatomic interactions. The NEGF + DFTB method is an accurate and efficient method for calculating transport properties and has been applied successfully to various nanostructured systems. ${ }^{23-25}$ The DFTB parameters for carbon and copper have been validated and reported earlier. ${ }^{26}$ The steady-state electrical current through the device under non-equilibrium conditions, i.e. for a finite bias voltage $\left(V_{\text {Bias }}\right)$ is calculated using the Landauer formula: ${ }^{27}$

$$
I\left(V_{\text {Bias }}\right)=\frac{2 e}{h} \int_{-\infty}^{+\infty} T\left(\varepsilon, V_{\text {Bias }}\right)\left[f\left(\varepsilon-\mu_{\mathrm{L}}\right)-f\left(\varepsilon-\mu_{\mathrm{R}}\right)\right] \mathrm{d} \varepsilon,
$$

where $f$ is the Fermi-Dirac distribution function, $\mu_{\mathrm{L}, \mathrm{R}}=$ $E_{\mathrm{F}} \pm e V_{\text {Bias }} / 2$ represent the chemical potentials of the left and right electrodes and $T\left(\varepsilon, V_{\text {Bias }}\right)$ is the energy and voltage-resolved transmission function. Electron transport is assumed to be ballistic since the electron mean free path in copper $(\sim 40 \mathrm{~nm})$ is much greater than the supercell dimensions. The calculations of the current are performed at $300 \mathrm{~K}$. For reference the bulk atomic and electronic structures of copper and graphene calculated with the DFTB method have been compared with DFT calculations and experimental data and the agreement is good (see Table S2 and Fig. S1, ESI $\dagger$ ).

Fig. 2 compares the current-voltage characteristics for composites with different chirality and orientation for models containing $2.87 \times 10^{13} \mathrm{~cm}^{-2}$ CNTs. Also shown is the characteristic of pure $\mathrm{Cu}$. Note that inelastic scattering effects, e.g. electronphonon coupling, have been ignored in the transport model.

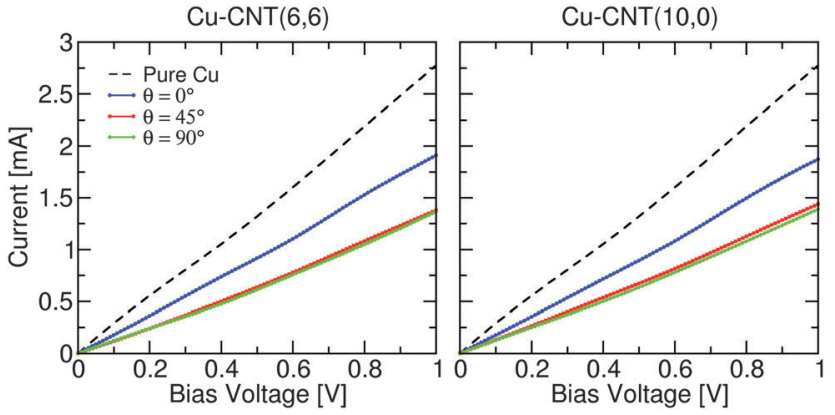

Fig. 2 Current-voltage characteristics of $\mathrm{Cu}-\mathrm{CNT}$ composites with different chirality and orientation. The characteristic of infinite pure $\mathrm{Cu}$ is also shown for comparison. 
However, this approximation does not change the general trends and conclusions obtained here, as the relative change in the conductance is not affected. It is seen that the presence of a CNT within the $\mathrm{Cu}$ matrix reduces the conductance (slope of the IV curve) by $\sim 30 \%$ in all cases compared to the intrinsic conductance of the copper. Noticeably the current through the composites is suppressed with increasing $\theta$. These results are consistent with previous reports that show that the end rather than the side of the CNT is the more favourable electron injection path. ${ }^{14,15}$ The effect of chirality on the conductance, at least for this CNT density, is very small and therefore the rest of the paper will focus on results for composites containing metallic $(6,6)$ CNTs.

In order to understand how the surface interactions between $\mathrm{Cu}$ and the CNT affect the transport properties, the electron difference density and electrostatic difference potential for the three $(6,6)$ CNT orientations are computed (see Fig. 3). The electrostatic difference potential is obtained by solving the Poisson equation where the charge density is taken from the electron difference density. The dark and light areas on the map correspond to electron loss and electron gain, respectively. The charge accumulation is mainly concentrated at the ends of each CNT while there is only slight charge transfer along the side contacts. This agrees well with previous studies on $\mathrm{Cu} / \mathrm{CNT}$ interfaces. ${ }^{15}$ It can be seen that the presence of the interface causes energy level re-alignment and the formation of a potential barrier, which can quench the electron transport. This evident from the two sharp peaks in the electrostatic difference potential which appear at $c=\sim 6 \AA$ and $\sim 17 \AA$ for the composite where the CNT is aligned along the bias direction. For the composite with oblique CNT alignment $\left(\theta=45^{\circ}\right)$, the two end effects overlap because of the shortness of the nanotube and only one peak is observed which decays smoothly along the transport direction.

Fig. 4(a) shows the projected density of states (PDOS) for the $\mathrm{Cu}-\mathrm{CNT}(6,6)$ composite with $\theta=0^{\circ}$. Due to the strong metallic character of the $\mathrm{Cu}$, the electronic structure close to the Fermi level is mostly governed by $\mathrm{Cu}$ d orbitals, while $\mathrm{C}$ atoms have no significant impact on the total DOS. Because of the dominance

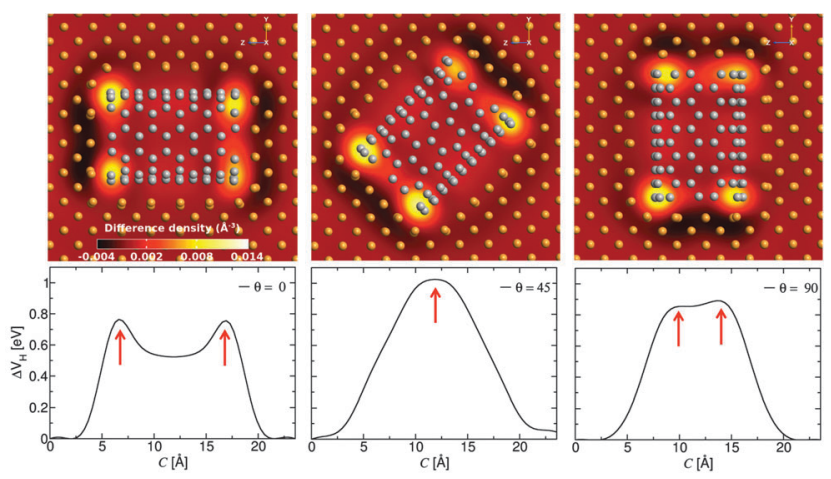

Fig. 3 Electron difference density maps through cross-sections of the scattering region (upper) and the average electrostatic difference potential along the transport direction (lower) for the three $\mathrm{Cu}-\mathrm{CNT}(6,6)$ composite orientations considered. The peak positions are indicated with red arrows to highlight the good correspondence between the upper and lower panel.
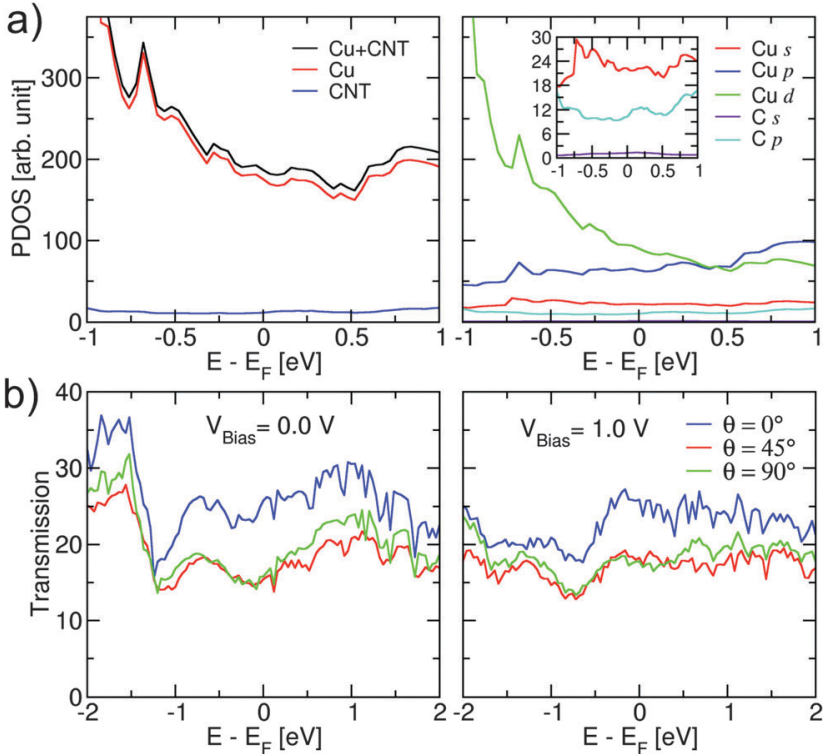

Fig. 4 (a) Projected density of states (PDOS) of the Cu-CNT $(6,6) \mathrm{com}$ posite with $\theta=0^{\circ}$ (see Fig. 1). The inset shows an enlarged view near the Fermi level. (b) Transmission coefficients as a function of energy at $V_{\text {Bias }}=$ $0.0 \mathrm{~V}$ and $V_{\text {Bias }}=1.0 \mathrm{~V}$ for $\mathrm{Cu}-\mathrm{CNT}(6,6)$ composites with different values of $\theta$ (see Fig. 1). The Fermi level $E_{\mathrm{F}}$ is shifted to zero.

by $\mathrm{Cu}$, the orientation of the CNT does not have a significant effect on the total density of states. Further DFT results, shown in Fig. S2 (ESI $\dagger$ ), on a $\mathrm{Cu} / \mathrm{CNT}$ interface indicate that for the end contact the cohesion between the CNT and copper surface is mainly due the coupling between carbon p orbitals and copper $\mathrm{d}$ orbitals. For the side contact, some slight $\mathrm{sp}^{2} / \mathrm{sp}^{3}$ re-hybridization of carbon atoms at the interface is seen. The calculated transmission coefficients of the composites for different values of $\theta$ and bias voltage are shown in Fig. 4(b). The results indicate a strong dependence of the transmission coefficient on the CNT orientation. The transmission value at the Fermi level is highest for $\theta=0^{\circ}$, while it decreases by about $40 \%$ for $\theta=45^{\circ}$ and $\theta=90^{\circ}$. The fewer available transmission pathways for electrons to flow from the $\mathrm{Cu}$ surface to the CNT can rationalize this effect. The overall transmission features are similar for the three cases and they indicate completely delocalized transport channels within the energy range shown here. The transmission coefficients at the Fermi energy are hardly affected by the bias voltage. However, for electron energies lower than the Fermi energy an increase in bias to $V_{\text {Bias }}=1.0 \mathrm{~V}$ leads to a significant decrease in the transmission coefficients. This suggests that electron transport through the composite is much stronger at lower bias than at higher bias. A similar behaviour is observed for composites with a $(10,0) \mathrm{CNT}$ (see Fig. S3 and S4, ESI $\dagger$ ). The SCC-DFTB transport results also showed very good agreement with DFT results obtained for $\mathrm{Cu}-\mathrm{CNT}$ composites with the same chirality and orientation (see Fig. S5, ESI $\dagger$ ).

To further investigate the transport behaviour in the composite, a comparison is made between the transmission eigenstates and pathways for pure $\mathrm{Cu}$ and the $\mathrm{Cu}-\mathrm{CNT}(6,6)$ composite with the most favourable orientation, $\theta=0^{\circ}$ (see Fig. 5). The results show that the presence of the CNT significantly changes the 

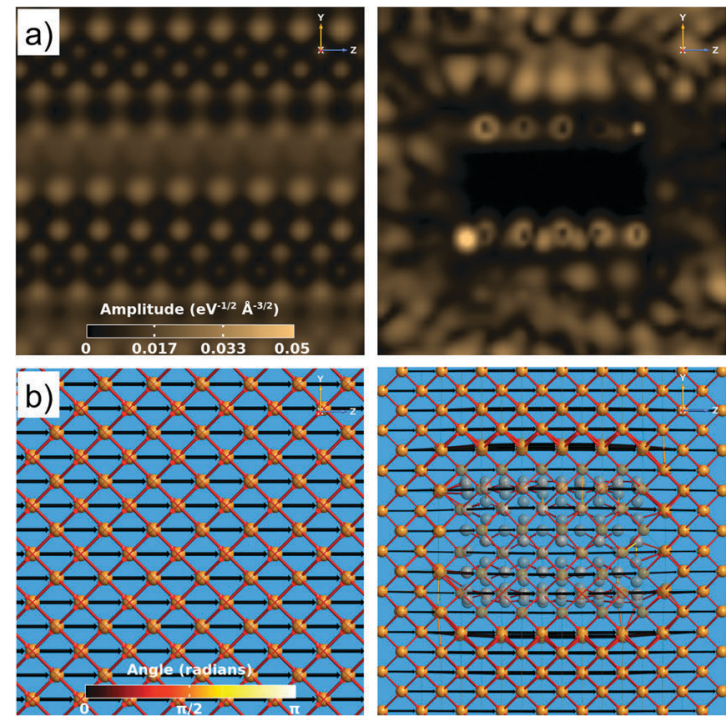

Fig. 5 (a) Transmission eigenstates and (b) transmission pathways for pure $\mathrm{Cu}$ (left) and a $\mathrm{Cu}-\mathrm{CNT}(6,6)$ composite with $\theta=0^{\circ}$ (right).

transmission modes in the composite particularly close to the $\mathrm{Cu} / \mathrm{CNT}$ interface. The amplitudes of the transmission coefficients are highest around the CNT circumference due to the excellent transport properties of CNT. In addition, the calculated transmission pathways show that some scattering occurs at the $\mathrm{Cu} / \mathrm{CNT}$ interface that can be responsible for the reduction in transmission through the composite.

Finally, the specific differential conductance $(\mathrm{d} I / \mathrm{d} V)$ of the $\mathrm{Cu}$-CNT $(6,6)$ composite with $\theta=0^{\circ}$ is calculated as a function of CNT number density per unit area and mass density of the composite (see Fig. 6). The highest CNT density per unit area considered in this work is about $5.1 \times 10^{13} \mathrm{~cm}^{-2}$. This value is consistent with recent theoretical ${ }^{18}$ and experimental data ${ }^{28}$ that report CNT densities around $10^{13}-10^{14} \mathrm{~cm}^{-2}$ for CNT-based composites. The calculated density of copper $\left(8.4 \mathrm{~g} \mathrm{~cm}^{-3}\right)$ is smaller than the expected value of $8.9 \mathrm{~g} \mathrm{~cm}^{-3}$ due to the $\sim 2.4 \%$ larger DFTB-predicted lattice constant compared to experiment (see Table S2, ESI $\dagger$ ). It is seen that conductance decreases with CNT number density and increases with overall mass density of the composite as expected. So, for example, a $\sim 10 \%$ reduction in mass density results in a $\sim 30 \%$ reduction in conductance.
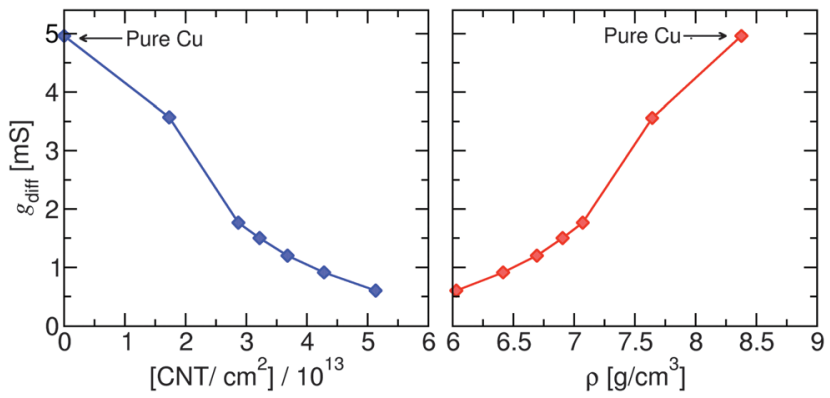

Fig. 6 Differential conductance of the $\mathrm{Cu}$-CNT $(6,6)$ composite with $\theta=$ $0^{\circ}$ as a function of CNT number density per unit area (left) and overall mass density of the composite (right).

\section{Conclusions}

Using the non-equilibrium Green's function approach, the transport properties of a $\mathrm{Cu}-\mathrm{CNT}$ composite are determined as a function of CNT density, chirality and alignment to the bias potential. A model is used in which the CNT is fully embedded within the $\mathrm{Cu}$ matrix. The electrical conductance is found to be highest when the axial direction of the CNT is along the transport direction. Compared to pure $\mathrm{Cu}$ the reduced conductance is attributed to localized charge exchange between the CNT and the metal surface that raises the potential barrier at the interface. The chirality of the CNT does not appear have a major effect on the conductance at least for the CNTs and densities considered here. The results could play an important role in guiding the design and fabrication of $\mathrm{Cu}-\mathrm{CNT}$ composites for optimal performance. Understanding and controlling the interface between the $\mathrm{Cu}$ and $\mathrm{CNT}$ in the bulk composite is critical for maximising the electrical performance of this promising new generation of materials.

\section{Acknowledgements}

The European Research Council provided financial support for this work under the Seventh Framework Program FP7/2007-2013 (ERC grant agreement no. 259061). Computational support from the Cambridge High Performance Computing Cluster is gratefully acknowledged.

\section{Notes and references}

1 B. Q. Wei, R. Vajtai and P. M. Ajayan, Reliability and Current Carrying Capacity of Carbon Nanotubes, Appl. Phys. Lett., 2001, 79, 1172-1174.

2 C. T. White and T. N. Todorov, Carbon Nanotubes as Long Ballistic Conductors, Nature, 1998, 393, 240-242.

3 J.-P. Salvetat, G. A. D. Briggs, J.-M. Bonard, R. R. Bacsa, A. J. Kulik, T. Stöckli, N. A. Burnham and L. Forró, Elastic and Shear Moduli of Single-Walled Carbon Nanotube Ropes, Phys. Rev. Lett., 1999, 82, 944-947.

4 M.-F. Yu, O. Lourie, M. J. Dyer, K. Moloni, T. F. Kelly and R. S. Ruoff, Strength and Breaking Mechanism of Multiwalled Carbon Nanotubes Under Tensile Load, Science, 2000, 287, 637-640.

5 T. W. Tombler, C. W. Zhou, L. Alexseyev, J. Kong, H. J. Dai, L. Lei, C. S. Jayanthi, M. J. Tang and S. Y. Wu, Reversible Electromechanical Characteristics of Carbon Nanotubes under Local-Probe Manipulation, Nature, 2000, 405, 769-772.

6 N. Behabtu, C. C. Young, D. E. Tsentalovich, O. Kleinerman, X. Wang, A. W. K. Ma, E. A. Bengio, R. F. ter Waarbeek, J. J. de Jong, R. E. Hoogerwerf, S. B. Fairchild, J. B. Ferguson, B. Maruyama, J. Kono, Y. Talmon, Y. Cohen, M. J. Otto and M. Pasquali, Strong, Light, Multifunctional Fibers of Carbon Nanotubes with Ultrahigh Conductivity, Science, 2013, 339, 182-186. 
7 A. Lekawa-Raus, J. Patmore, L. Kurzepa, J. Bulmer and K. Koziol, Electrical Properties of Carbon Nanotube Based Fibers and Their Future Use in Electrical Wiring, Adv. Funct. Mater., 2014, 24, 3661-3682.

8 L. Kurzepa, A. Lekawa-Raus, J. Patmore and K. Koziol, Replacing Copper Wires with Carbon Nanotube Wires in Electrical Transformers, Adv. Funct. Mater., 2014, 24, 619-624.

9 Y. Cho, C. Kim, H. Moon, Y. Choi, S. Park, C.-K. Lee and S. Han, Electronic Structure Tailoring and Selective Adsorption Mechanism of Metal-Coated Nanotubes, Nano Lett., 2008, 8, 81-86.

10 S. R. Bakshi, D. Lahiri and A. Agarwal, Carbon Nanotube Reinforced Metal Matrix Composites - a Review, Int. Mater. Rev., 2010, 55, 41-64.

11 C. Subramaniam, T. Yamada, K. Kobashi, A. Sekiguchi, D. N. Futaba, M. Yumura and K. Hata, One Hundred Fold Increase in Current Carrying Capacity in a Carbon Nanotube-Copper Composite, Nat. Commun., 2013, 4, 2202.

12 C. Subramaniam, Y. Yasuda, S. Takeya, S. Ata, A. Nishizawa, D. Futaba, T. Yamada and K. Hata, Carbon NanotubeCopper Exhibiting Metal-like Thermal Conductivity and Silicon-like Thermal Expansion for Efficient Cooling of Electronics, Nanoscale, 2014, 6, 2669-2674.

13 G. Chai, Y. Sun, J. Sun and Q. Chen, Mechanical Properties of Carbon Nanotube-Copper Nanocomposite, J. Micromech. Microeng., 2008, 18, 035013.

14 Y. Matsuda, W.-Q. Deng and W. A. Goddard, Contact Resistance for "End-Contacted" Metal-Graphene and Metal-Nanotube Interfaces from Quantum Mechanics, J. Phys. Chem. C, 2010, 114, 17845-17850.

15 F. Gao, J. Qu and M. Yao, Electronic Structure and Contact Resistance at an Open-End Carbon Nanotube and Copper Interface, Appl. Phys. Lett., 2010, 96, 102108.

16 G. Wu, Q. Meng and C. Wang, I-V Curves of Carbon Nanotubes with Copper Contacts Using Non-Equilibrium Green's Function Method, Phys. E, 2011, 44, 146-151.

17 A. Zienert, J. Schuster and T. Gessner, Metallic Carbon Nanotubes with Metal Contacts: Electronic Structure and Transport, Nanotechnology, 2014, 25, 425203.
18 Y. Feng and S. L. Burkett, Modeling a Copper/carbon Nanotube Composite for Applications in Electronic Packaging, Comput. Mater. Sci., 2015, 97, 1-5.

19 G. Seifert, D. Porezag and T. Frauenheim, Calculations of Molecules, Clusters, and Solids with a Simplified LCAO-DFT-LDA Scheme, Int. J. Quantum Chem., 1996, 58, 185-192.

20 Atomistix ToolKit 2014, QuantumWise A/S, www.quantum wise.com.

21 S. Datta, Quantum Transport: Atom to Transistor, Cambridge University Press, New York, 2nd edn, 2005.

22 M. Elstner, D. Porezag, G. Jungnickel, J. Elsner, M. Haugk, T. Frauenheim, S. Suhai and G. Seifert, Self-ConsistentCharge Density-Functional Tight-Binding Method for Simulations of Complex Materials Properties, Phys. Rev. B: Condens. Matter Mater. Phys., 1998, 58, 7260.

23 M. Ghorbani-Asl, S. Borini, A. Kuc and T. Heine, StrainDependent Modulation of Conductivity in Single-Layer Transition-Metal Dichalcogenides, Phys. Rev. B: Condens. Matter Mater. Phys., 2013, 87, 235434.

24 M. Ghorbani-Asl, N. Zibouche, M. Wahiduzzaman, A. F. Oliveira, A. Kuc and T. Heine, Electromechanics in $\mathrm{MoS}_{2}$ and $\mathrm{WS}_{2}$ : Nanotubes vs. Monolayers, Sci. Rep., 2013, 3, 2961.

25 R. Juarez-Mosqueda, M. Ghorbani-Asl, A. Kuc and T. Heine, Electromechanical Properties of Carbon Nanotubes, J. Phys. Chem. C, 2014, 118, 13936-13944.

26 B. Lukose, B. Supronowicz, P. St. Petkov, J. Frenzel, A. B. Kuc, G. Seifert, G. N. Vayssilov and T. Heine, Structural Properties of Metal-Organic Frameworks within the DensityFunctional Based Tight-Binding Method, Phys. Status Solidi, 2012, 249, 335-342.

27 R. Landauer, Spatial Variation of Currents and Fields due to Localized Scatterers in Metallic Conduction, IBM J. Res. Dev., 1957, 1, 223-231.

28 J. Robertson, G. Zhong, S. Hofmann, B. C. Bayer, C. S. Esconjauregui, H. Telg and C. Thomsen, Use of Carbon Nanotubes for VLSI Interconnects, Diamond Relat. Mater., 2009, 18, 957-962. 